\title{
Absolute polarimeter APol for NICA collider project
}

\section{Mikhail Kulikov ${ }^{1}$}

Joint Institute for Nuclear Research

141980 Moscow region, Dubna, Joliot-Curie 6, Russia

E-mail: mkulikovajinr.ru

\section{Victor Fimushkin}

Joint Institute for Nuclear Research

141980 Moscow region, Dubna, Joliot-Curie 6, Russia

E-mail: fimushkinejinr.ru

\section{Roman Kuzyakin}

Joint Institute for Nuclear Research

141980 Moscow region, Dubna, Joliot-Curie 6, Russia

E-mail: kuzyakinejinr.ru

\section{Yuriy Prokofichev}

Joint Institute for Nuclear Research

141980 Moscow region, Dubna, Joliot-Curie 6, Russia

E-mail: yuriyejinr.ru

\section{Alexey Shumkov}

Joint Institute for Nuclear Research

141980 Moscow region, Dubna, Joliot-Curie 6, Russia

E-mail: ashumkoffejinr.ru

\begin{abstract}
A conceptual design of the absolute polarimeter APol with an internal polarized atomic hydrogen/deuterium jet target for NICA collider is presented. It is proposed to install the polarimeter into the "warm" gap of the collider ring arc. The polarized jet will cross the both accelerated beams. The jet target is based on the classical atomic beam source principle. The expected target thickness of the jet in the interaction regions is $10^{12} \mathrm{atom} / \mathrm{cm}^{2}$. Polarization of the atomic hydrogen/deuterium jet will be measured by Breit-Rabi polarimeter placed under the collider ring into the jet catcher volume. APol will utilize a reaction of elastic scattering of identical nuclei (protons or deuterons).
\end{abstract}

23rd International Spin Physics Symposium - SPIN2018 -

10-14 September, 2018

Ferrara, Italy

\footnotetext{
${ }^{1}$ Speaker

(C) Copyright owned by the author(s) under the terms of the Creative Commons 


\section{Introduction}

To measure absolute values and signs of proton or deuteron polarization in the NICA collider rings the absolute polarimeter APol is being built. It is proposed to install the polarimeter into the "warm" gap of the collider ring arc. The polarized jet will cross the both accelerated beams. The jet target is based on the classical atomic beam source principle. The expected target thickness of the jet in the interaction regions is $10^{12}$ atom $/ \mathrm{cm}^{2}$. Polarization of the atomic hydrogen/deuterium jet will be measured by Breit-Rabi polarimeter placed under the collider ring into the jet catcher volume. APol will utilize a reaction of elastic scattering of identical nuclei (protons or deuterons).

\section{Polarimetric reaction and polarization measurement basics}

These measurements are made by means of comparison of the recoil particle registration asymmetry in scattering the accelerated unpolarized beam on polarized nuclei of atomic hydrogen or deuterium and of the recoil particle registration asymmetry in scattering the accelerated polarized beam on unpolarized nuclei of atomic hydrogen or deuterium. The measured beam polarization:

$P_{\text {beam }}=-\frac{\varepsilon_{\text {beam }}}{\varepsilon_{\text {jet }}} P_{\text {jet }}=-\frac{\varepsilon_{\text {beam }}}{A_{N}}$,

where

$$
A_{N}=\frac{\varepsilon_{j e t}}{P_{j e t}}
$$

- the analizing power of the polarimetric reaction, and

$$
\varepsilon \equiv \frac{N_{\text {left }}-N_{\text {right }}}{N_{\text {left }}+N_{\text {right }}}
$$

- the measured assymetry.

Reactions of elastic scattering of protons on hydrogen nuclei and deuterons on deuterium nuclei were chosen as polarimetric reactions. $200 \mathrm{MeV}$ recoil particles will be registered under 75 degrees in the lab system. In the accelerated beam energy range from $3 \mathrm{GeV}$ to $11 \mathrm{GeV}$ the analyzing power of these reactions is in the range from $20 \%$ to $8 \%$.

\section{Actual geometry requirements}

One of the specific features of NICA collider is positioning of two lines of the beam acceleration and transportation parts one under another at a distance of $32 \mathrm{~cm}$. The hydrogen and deuterium atoms with polarized nuclei used as a target in the polarimetric reaction, are combined into the directed atomic beam within a part of the polarimeter named Atomic Beam Source (ABS). The atomic beam crosses the both accelerated beams (See Fig.1). It means that the atomic beam axis is normal to the ring planes and then the detector arms must be directed in the ring planes and the axes of polarization of the beams in the interaction regions must be directed along the atomic beam.

\section{Mesuring time estimates}

It seems useful to estimate the time for measuring by means of APol. Details of the estimations are presented to show which factors influence the measuring time. For example, due to a smaller 
perimeter of the ring the NICA collider has about 8 times a higher repetition rate of crossing the jet by accelerated particles than at RHIC, (Brookhaven, USA).

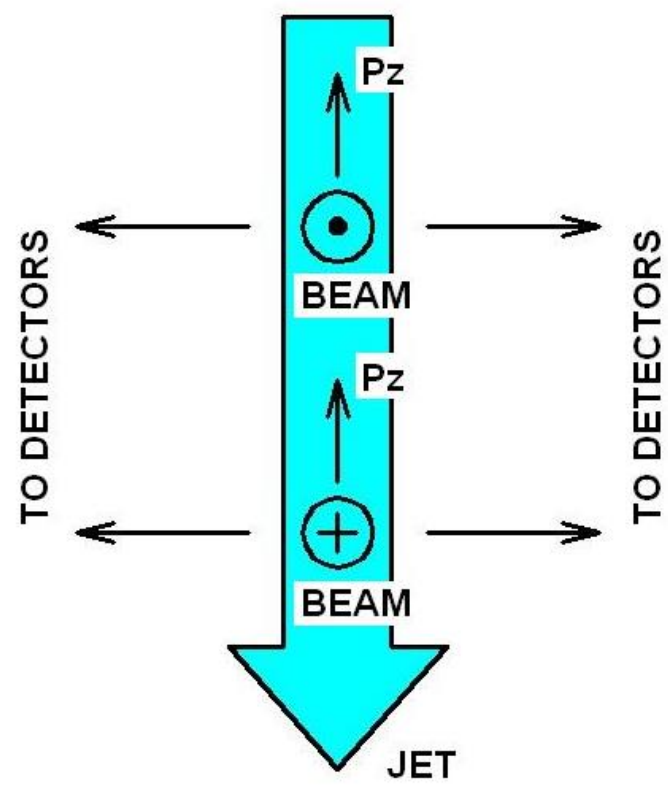

Figure 1: Actual geometry requirements.

The expected rate of data acquisition:

$N=\Delta \sigma \cdot L \cdot \Delta \psi$,

where :

$\Delta \sigma$ - particle scattering cross-section into the direction of registration,

$\Delta \psi(=0.016)$ - a relative solid angle into the direction of registration,

$L$ - luminosity

In its turn:

$L=N_{\text {bunch }} \cdot n_{\text {bunch }} \cdot F \cdot t_{\text {jet }}$,

where:

$N_{\text {bunch }}\left(=10^{12}\right)$ - the number of protons/deuterons per bunch (for NICA)

$n_{\text {bunch }}(=22)-$ the number of bunches (for NICA)

$F\left(=3 \cdot 10^{8} \mathrm{~m}^{*} \mathrm{~s}^{-1} / 503 \mathrm{~m}=6 \cdot 10^{5} \mathrm{~s}^{-1}\right)$ - frequency of crossing the jet by accelerated particles (for

RHIC it is $3.8 / 0.5=7.6$ times smaller)

$t_{\text {jet }}\left(=10^{12}\right.$ atom $\left./ \mathrm{cm}^{2}\right)-$ target thickness of the jet,

and numerically:

$L=10^{12} \cdot 22 \cdot 6 \cdot 10^{5} \cdot 10^{12}=1.3 \cdot 10^{31} \mathrm{~s}^{-1} \cdot \mathrm{cm}^{-2}=1.3 \cdot 10^{4} \mathrm{~s}^{-1} \cdot \mathrm{mb}^{-1}$

The number of the events needed to measure $\mathrm{A}_{\mathrm{N}}$ with the accuracy of $5 \%$ :

$N_{A}=\left(0.05 \cdot A_{N}\right)^{-2}$

The time needed to measure $A_{N}$ with the accuracy of $5 \%$ :

$T=N_{A} / N$

If we put numbers into the table, we will see the following: 


\begin{tabular}{|c|c|c|c|c|}
\hline $\begin{array}{c}\text { Energy of } \\
\text { the beam, } \\
\mathrm{GeV}\end{array}$ & $\begin{array}{c}\text { Scattering cross- } \\
\text { section, mb }\end{array}$ & $\begin{array}{c}\text { Analizing } \\
\text { power }\end{array}$ & $\begin{array}{c}\text { Number of events } \\
\text { needed for } \\
\delta \mathrm{A}_{\mathrm{N}}=5 \%\end{array}$ & $\begin{array}{c}\text { Time of } \\
\text { measurement }\end{array}$ \\
\hline 3 & 3.08 & 0.195 & 10500 & 18 seconds \\
\hline 7 & 2.29 & 0.107 & 35000 & 73 seconds \\
\hline 11 & 1.84 & 0.077 & 67500 & 182 seconds \\
\hline
\end{tabular}

Data for cross-sections and analizing powers are taken from [1].

\section{Placement of APol at NICA collider}

APol needs about 1 meter of the NICA ring (See Fig.2). It is better to install the polarimeter into the "warm" gap near the beam injection into the ring because of the space quite enough for the walls of the NICA building. For example, on the SPD side as it is shown in Figure 2.

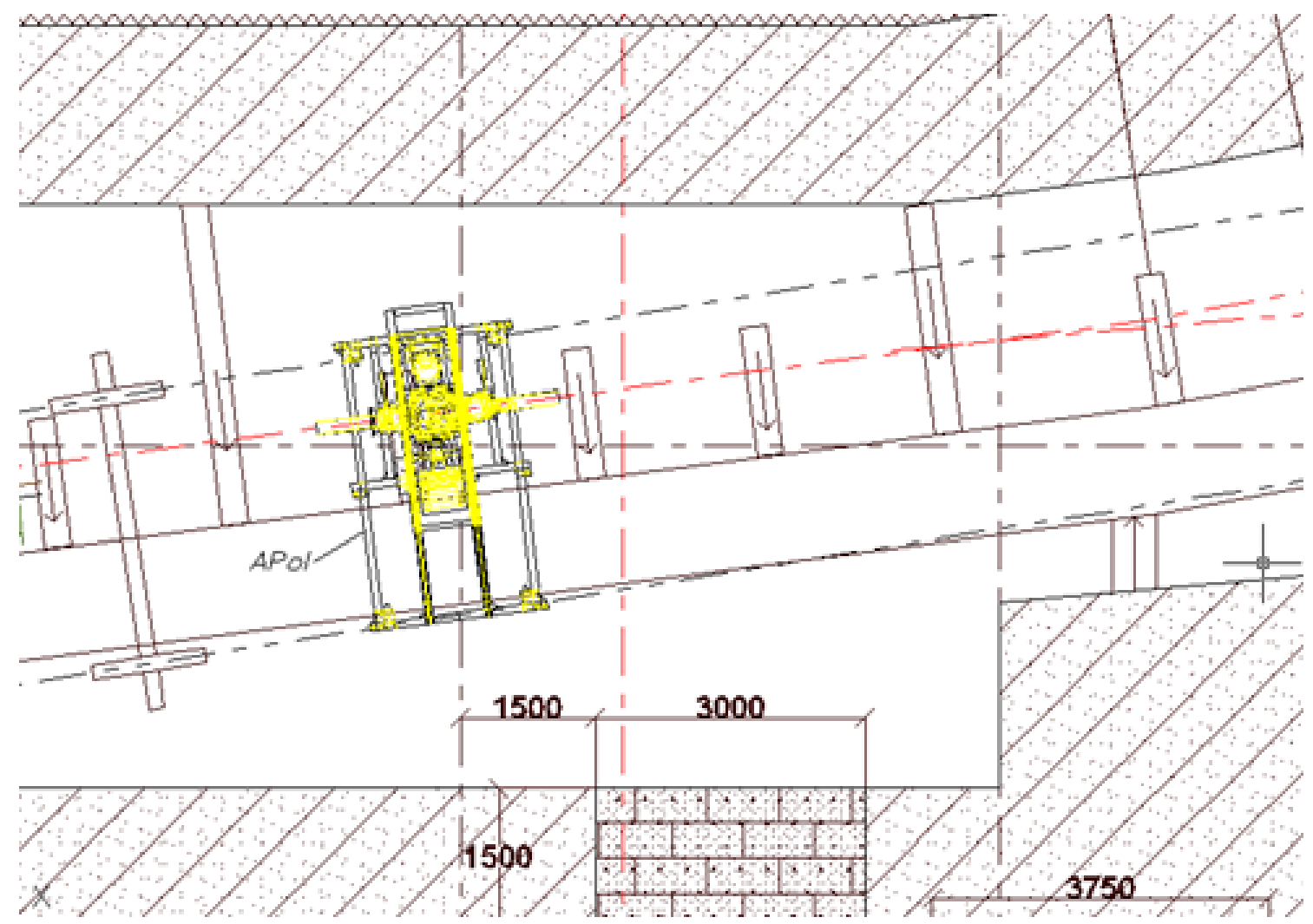

Figure 2: Placement of APol at NICA collider

\section{Design and main dimensions of APol}

APol consists of the following large subunits (See Fig.3):

- Atomic Beam Source - ABS,

- Interaction box,

- Spectrometer arms - four units

- Jet catcher with a Breit-Rabi polarimeter

- Polarimeter frame with movable and fixed parts 


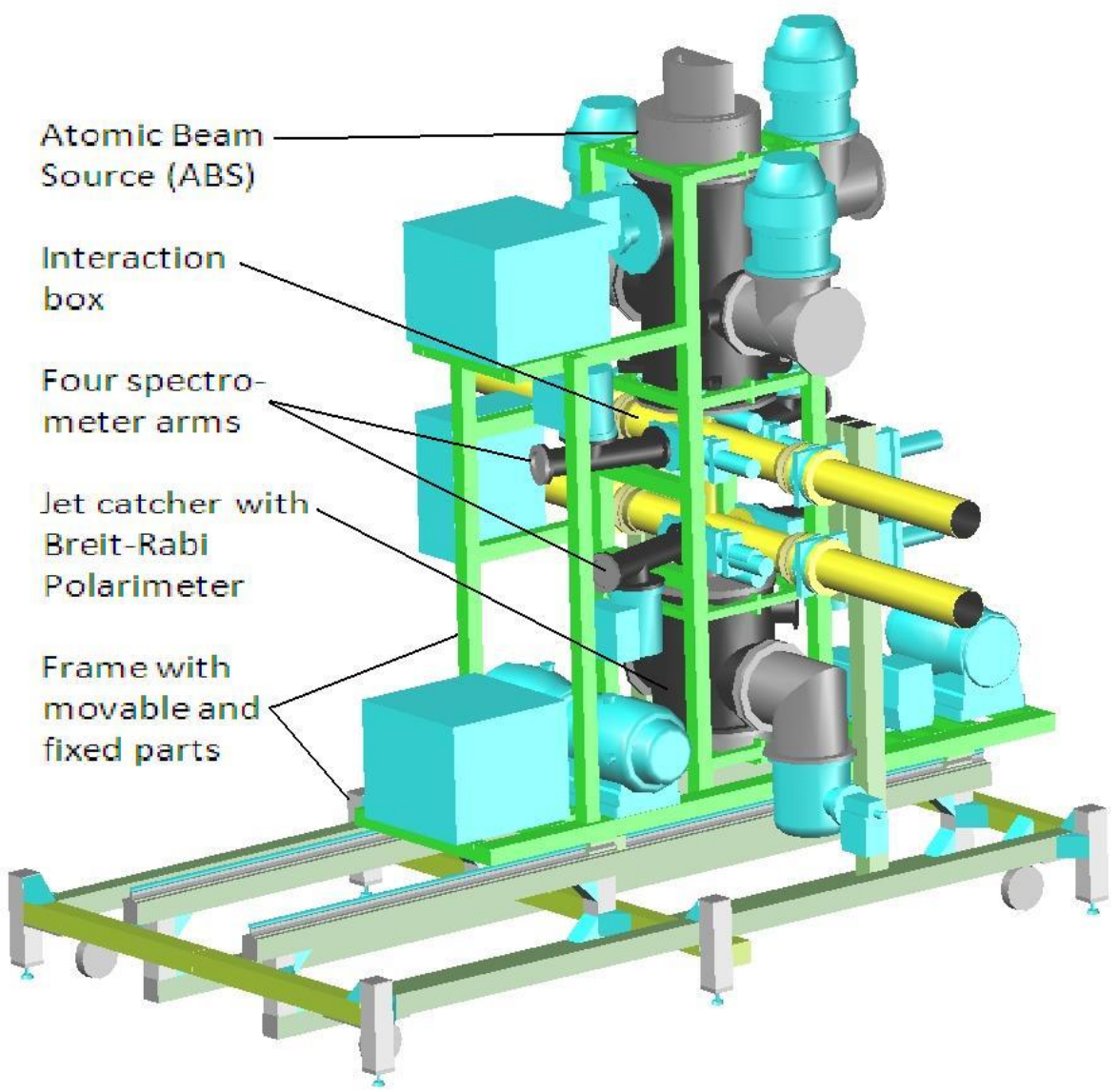

Figure 3: Main subunits of APol.

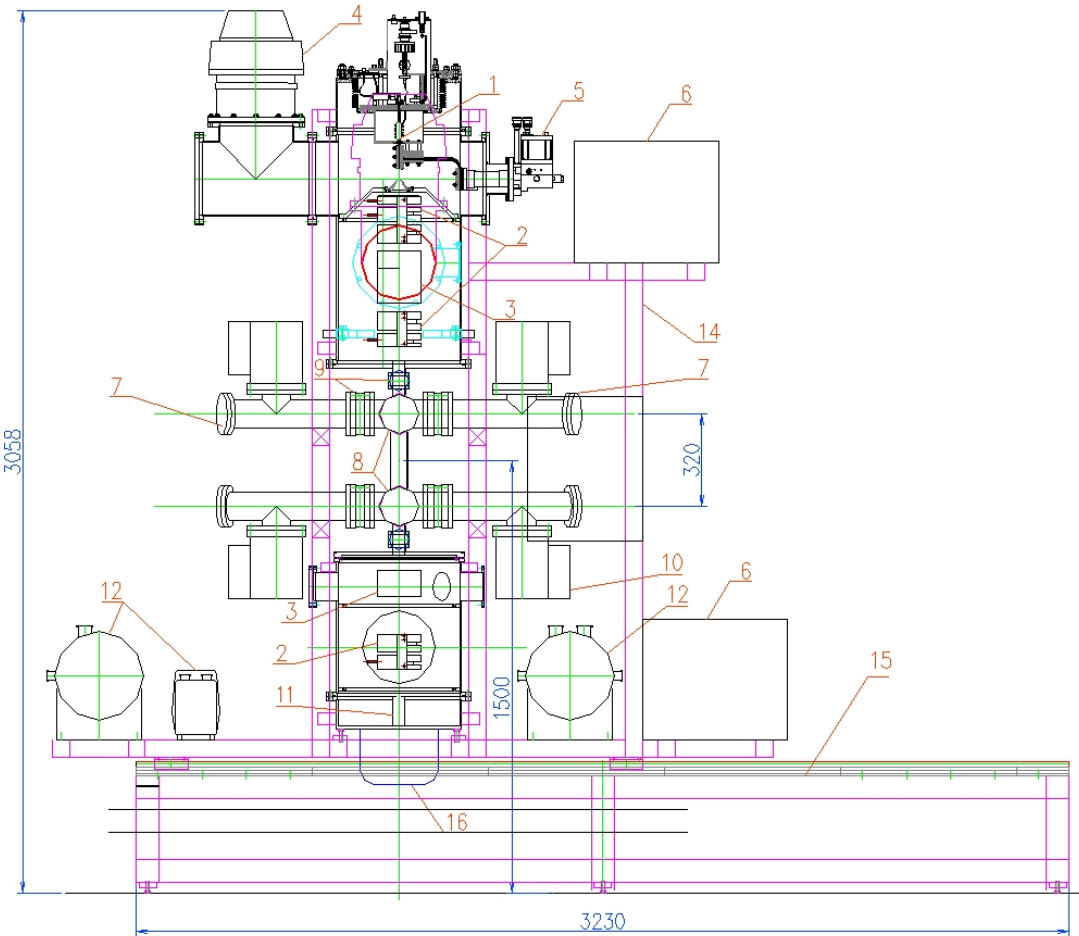

Figure 4: APol scheme.
Parts of APol:

1. Dissociator

2. Sextupole magnets

3. Nuclear polarization cells

4.Turbomolecular pumps $1850 \mathrm{l} / \mathrm{s}$

5. Cryocooler $(78 \mathrm{~K})$

6. Cryocooler compressor

7. Detector arm

8. Interaction box

9. UHV valves

10.Turbomolecular pumps $4501 / \mathrm{s}$

11. Massspectrometer

12. Forepumps

14. Movable frame

15. Fixed frame

16. Cryopump $3200 \mathrm{l} / \mathrm{s}$ 
The interaction box is connected to the beampipes by Ultra High Vacuum valves and bellows that allows one to cut off the box from the beampipes and shift it together with the entire polarimeter transversely to the beams. The setup operates in a continuous mode. The separation of the beam atoms due to the spin orientation of their electrons and focusing onto the interaction regions is made by a set of six permanent Nd-Fe-B sextupole magnets. Each magnet is combined of 24 wedge segments. The expected target thickness of the jet in the interaction regions is $10^{12}$ atom $/ \mathrm{cm}^{2}$.

\section{Conclusion}

The polarimeter APol allows to carry out fast (few minutes) measurements of absolute polarization values and signs of proton and deuteron accelerated beams. The measurements can be made simultaneously on the both NICA beams by using a single polarized jet target. The polarimeter APol requires $1 \mathrm{~m}$ of the NICA rings in the "warm" gap near the beam injection regions and the beams polarization axes must be normal to the ring planes in the interaction regions.

\section{References}

[1] H. Spinka et al, Beam polarization at the ZGS, NIMA 211 (1983) 239-261. 